\title{
Camas drenantes bajo las líneas de drenaje de la zona metropolitana de Guadalajara (ZMG)
}

\section{Drainage beds under the drain lines Guadalajara metropolitan area (GMA)}

\author{
Carlos Alejandro Vázquez Ortiz \\ Ingeniero Civil, magíster en Mecánica de Suelos. Docente de la Universidad de \\ Guadalajara. Guadalajara, México. Contacto: c_alejandro_vo@hotmail.com.

\section{Carlos Gregorio Pastrán Beltrán} \\ Ingeniero Civil, estudiante de Doctorado. Docente Asistente de la Universidad \\ Distrital Francisco José de Caldas. Bogotá, Colombia. \\ Contacto: cgpastranb@udistrital.edu.co.
}

Fecha de recepción: 30 de enero de 2013

Clasificación del artículo: investigación

Fecha de aceptación: 15 de abril de 2013

Financiamiento: Instituto de Ingeniería Sísmica de la Universidad de Guadalajara

Palabras clave: arena, colapso, drenaje.

Key words: sand, collapse, drainage.

\section{RESUMEN}

En este artículo se presenta un procedimiento constructivo modificado para las líneas de drenaje de interiores, así como de la red municipal. Forma parte de un problema que aqueja las vialidades y edificaciones de la Zona Metropolitana de Guadalajara ZMG; que es la falla en su estructura debido a socavones en el suelo de apoyo, provocado por fugas de agua en las líneas de drenaje que derivan en la tubificación de suelo por la presión que origina el flujo de agua, debido a la saturación del suelo de apoyo y consecuente disminución en su resistencia. Se propone la inclusión de una cama drenante construida de una capa de arena pumítica común en la mayor parte del área que comprende la ZMG. Este material tiene alta permeabilidad y buena resistencia si es compactada. Se incluye la instalación de rejillas a determinada distancia para evitar el arrastre de finos por tubificación. Se incluye además una capa de polietileno en la base que impida la filtración de agua al suelo de apoyo.

\section{ABSTRACT}

This paper presented a modified construction procedure for interior drainage lines and munici- 


\section{investigación}

pal network. One of the problems that affect the roads and buildings of the GMA is the failure of this structure due to tunnels in the ground support. This is caused by water leaks in the drains lines due to the water pressure flow that came from the soil piping due to the saturation of the ground support and consequently decreasing the resistance.

In this paper it has been proposed the inclusion of a drainage bed, composed of a layer of pum- itic sand, which could compromise the main GMA. This material develops high permeability and good resistance if it is compacted. Also, it has been included the installation of grids at certain distance to avoid the loss of fine particles due to piping. Moreover, a polyethylene layer has been added to the drainage bed, which prevents water leaking thought the ground support.

\section{INTRODUCCIÓN}

Los sistemas para la recolección y tratamiento de aguas residuales y agua potable, diseñados con criterios convencionales, demandan elevados costos de construcción y operación.

El adecuado drenaje es esencial para evitar la destrucción total o parcial de un camino o una construcción, y reducir impactos indeseables al ambiente debido a la escorrentía a lo largo de las líneas.

Los principios que orientan el desarrollo de un buen sistema de drenaje son elementales y requieren gran atención en la etapa de proyecto. Deben tenerse en cuenta los factores climáticos, topográficos geológicos y el tipo de suelo.

La Zona Metropolitana de Guadalajara (ZMG) comprende los municipios de Guadalajara, El Salto, Juanacatlán, Ixtlahuacan de los membrillos, Tlajomulco de Zuñiga, Tlaquepaque y Tonalá, Zapopán.

El subsuelo de Guadalajara está constituido, básicamente, por depósitos de fragmentos de piedra pómez cuyo material varía entre arena y grava. Existen gravas de material pumítico del orden de 1 a $9 \mathrm{~cm}$ de diámetro y finas. También existen zonas de estas arenas pumíticas con finos de arcilla, las partículas de grava y arena se presentan de varias formas, texturas y colores de acuerdo al tipo de grano de la piedra pómez. Se tienen granos de forma irregular y semi-angulosos que van desde 2,2 a 7,6 cm. Otros depósitos en que los granos han sido acarreados y transportados por el agua o por el viento tienen forma redondeada $(1,5$ a 2,6 cm). La textura puede ser esponjosa con poros interiores visibles, filiforme o fibroso. En su color predomina el amarillo pero también se encuentran arenas de color blanco y café. Evidentemente estos productos están relacionados con la actividad volcánica de la caldera la primavera, por lo que nos estaremos refiriendo entonces a tobas pumíticas. Sin embargo, en la práctica del ingeniero civil en la ZMG, es común referirse en términos de sus diámetros más que en términos de su origen.

El objetivo de este trabajo es adecuar los procedimientos constructivos existentes para las líneas de drenaje, con la implementación de una cama drenante construida con material del lugar, que en caso de existir fuga conduzca el flujo de agua al siguiente registro o pozo de visita aguas abajo, evitando que el agua se filtre al suelo de apoyo y provoque el fenómeno de colapso por saturación que se presenta actualmente en la red de alcantarillado. 


\section{METODOLOGÍA}

Para plantear la modificación del sistema constructivo, primero se demostró cómo se presenta el fenómeno de tubificación y, posteriormente, las características que deberá tener la cama drenante para desalojar el flujo producto de una fuga en la línea de drenaje. Para ello la investigación se desarrollo en cinco fases descritas a continuación.

La primera fase fue la recopilación de la información actual, sobre suelos colapsables, su identificación y clasificación, así como los métodos para la determinación de colapso por saturación. La segunda fase fue la exploración del subsuelo con sondeos de pozo a cielo abierto PCA, la recuperación de muestras alteradas y muestra cúbica. La tercera fase fue identificar los estratos de suelo colapsable, realizando pruebas índice. contenido de agua, límites de consistencia -límite líquido, límite plástico, índice de plasticidad y contracción lineal-, peso específico, densidad de sólidos, granulometrías, peso volumétrico suelto y compacto, permeabilidad, pruebas de comprensión triaxíal. La cuarta fase consistió en la simulación del fenómeno de suelo colapsable, en un modelo a escala. La quinta y última fase fue el análisis de las características geotécnicas de la arena pumitica a emplear como dren debajo de las líneas de drenaje.

\section{RESULTADOS}

\subsection{Suelos colapsables.}

En la actualidad, en la ZMG encontramos fallas en la estructura de vialidades y edificaciones producto de socavones en el suelo de apoyo; esto, en la mayoría de los casos, es debido a filtraciones de agua originadas por fugas en las líneas de drenaje que provocan saturación del suelo y con ello disminución en su resistencia, lo cual ocasiona colapso y tubificación del suelo que provoca da- ños en las construcciones. Provocados por la mala colocación de la tubería, por la falta de mantenimiento y debido a que estas son apoyadas en arena amarilla -arena limosa con clasificación SUCS (SM)- susceptible al colapso por saturación bajo carga.

El estudio del comportamiento de los suelos colapsables es complejo y no siempre se produce por causas mecánicas, en otras ocasiones, intervienen diferentes factores -químicos, ambientales, entre otros- conocidos genéricamente como suelos estructuralmente inestables.

Uno de los principales fenómenos que afectan a algunos de estos suelos es el colapso brusco de su estructura intergranular, denominándose a los suelos que presentan estas características: suelos colapsables [1].

En muchos aspectos, los suelos colapsables pueden ser considerados de comportamiento opuesto al de los suelos expansivos. En ambos, la presión del agua de poro inicial es negativa. En ambos casos, los movimientos son el resultado de un incremento en la presión negativa del agua de poro. El humedecimiento de un suelo colapsable, sin embargo, resulta en un decremento de volumen. El colapso del suelo puede ocurrir tanto en un talud natural, como en un suelo de cimentación [2].

En los suelos parcialmente saturados con una estructura abierta, al aumentar el grado de saturación, debido a cambios ambientales o de otro tipo, se pueden producir reducciones volumétricas irrecuperables sin que varíen las solicitaciones externas del suelo; es decir, se produce el colapso sin aumento de la carga aplicada, a este fenómeno se le conoce con el nombre de colapso (figura 1).

Donde:

ab: determinada a partir del ensayo de consolidación para un espécimen a humedad natural. 


\section{investigación}

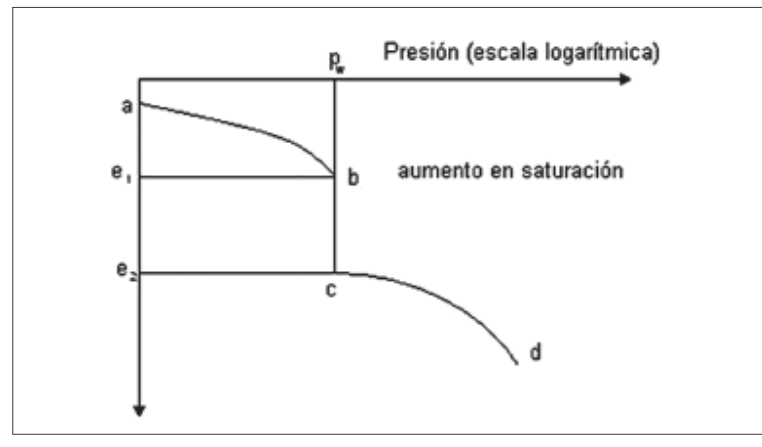

Figura 1. Fenómeno de colapso

Fuente: tomado de [3]

$\mathrm{e}_{1}$ : relación de vacíos equilibrio antes de saturación.

$\mathrm{e}_{2}$ : relación de vacíos equilibrio después de saturación.

cd: curva después de cargar adicionalmente, después de la consolidación.

En general, los suelos colapsables presentan una serie de características comunes, tales como [34]:

- Estructura macroporosa, con índice de huecos (e), entre relativamente alto, a muy alto.

- Granulometría predominantemente fina, con predominio de fracciones de limos y de arci1la. El tamaño de los granos es generalmente poco distribuido y con los granos más grandes escasamente meteorizados. La mayoría de las veces, la cantidad de la fracción arcilla es relativamente escasa, sin embargo, tiene una influencia importante en el comportamiento mecánico de la estructura intergranular.

- Estructura mal acomodada, con partículas de mayor tamaño, separadas por espacios abiertos, y unidas entre sí por acumulaciones o "puentes" de material predominantemente arcilloso. En muchos casos existen cristales de sales solubles insertados en tales puentes o uniones arcillosas.
En el suelo de análisis, el colapso es ocasionado por un incremento del contenido de humedad. Por lo tanto, el fenómeno provoca una brusca disminución de volumen, sin la necesidad de un aumento en la presión aplicada.

Por lo anterior, se advierte; por un lado, una destrucción o un cambio en la estructura que el suelo tenía originalmente; y por otro lado, un agente externo: el agua, que provoca este fenómeno.

Un suelo con tendencia al colapso, en general, es estable mientras el grado de saturación es inferior a cierto límite; a partir del cual la llegada del agua puede debilitar suficientemente los enlaces existentes como para que se produzcan deslizamientos tangenciales en los contactos entre partículas. Los enlaces entre partículas del suelo pueden ser de varios tipos [5]: 1) enlace capilar que se presenta fundamentalmente en los limos y arenas. Los meniscos que se forman en la interfase (aire-agua-partículas sólidas), generan fuerzas normales que aumentan los esfuerzos entre dichas partículas, rigidizando el conjunto. En el caso de las arcillas, este fenómeno no es tan claro a nivel de partículas, aunque es probable que ocurra a nivel de agrupaciones más grandes que estas; 2) en cualquier caso, si el grado de saturación crece por aumento de la humedad o por reducción del índice de vacíos, estos enlaces desaparecen, con lo que el conjunto se debilita pudiendo llegar al colapso si la presión exterior aplicada es suficientemente grande.

El colapso no se produce al mismo tiempo ni con la misma humedad en todos los tipos del suelo. Existen suelos más arenosos, donde el agua desestabiliza el esqueleto cuando se humedecen y se produce el colapso, pero los agregados no se rompen ni se fusionan entre si (figura 2).

Al humedecer una arena parcialmente saturada, se desestabiliza el sistema por efecto de la capilaridad al inundarla y se crea un pequeño colapso, 


\section{investigación}

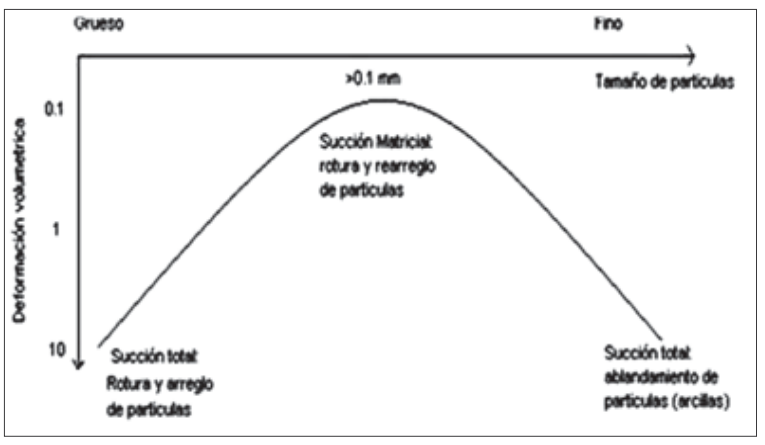

Figura 2. Colapso por humedecimiento

Fuente: tomado de [3]

pero como no hay esfuerzos tan grandes que rompan las partículas no hay fusión de estas, sino solo un reacomodo.

Los suelos con tendencia a presentar a partículas muy finas de tipo arcilloso, presentan un comportamiento seco aparentemente rígido, pero en presencia de agua lo pierden, permitiendo un reacomodo esqueletal y la deformación-fusión de las partículas, produciendo el colapso.

En cuanto a los tipos de métodos de identificación propiamente dichos, varios han sido los enfoques que se han propuesto [6]. Estos podrían clasificarse en tres grupos: 1) métodos basados en parámetros físicos de identificación de suelos, como: Peso Unitario, Límites de Consistencia, Granulometría, entre otros; 2) métodos basados en ensayos mecánicos, principalmente en ensayos edométricos y 3 ) métodos basados en la magnitud del colapso. Nuestro análisis se basa en el primer método descrito.

\section{Soluciones ingenieriles en suelos colapsables}

La primera cuestión que debe analizarse cuando se diseñan cimentaciones en suelos susceptibles al colapso, es la probabilidad que el agente desencadenante del fenómeno, el agua, pueda o no introducirse en el terreno y, por tanto, "sensibili- zar" al suelo en donde se apoyarán las estructuras. Por lo tanto, sin la presencia del agua, el suelo no colapsa.

Las soluciones a este problema, se pueden dividir en [7]:

a) Tratamiento del suelo colapsable con vista a eliminar la tendencia al colapso a lo largo de todo el estrato de suelos desmoronables.

b) Diseño de elementos constructivos que eliminen o disminuyan, a límites razonables, la posibilidad que se inicie el colapso.

c) Diseño de estructuras y cimentaciones insensibles a los asentamientos provocados por el colapso, por ejemplo, cimentaciones profundas apoyadas sobre un manto no sujeto a los asentamientos por humedecimiento.

d) Geomembranas.

e) Métodos de mejoramiento de las propiedades del suelo por drenaje.

\section{Tipos de tubificación}

En los primeros estudios sobre este fenómeno se analizaron "reventones" en los cuales la arena entra en "ebullición" y provoca el colapso de la obra si el peso del suelo es insuficiente para contener el movimiento ascendente del mismo, bajo el efecto de la fuerza de filtración, cuando se alcanza un determinado gradiente "crítico". Se recomenienda la construcción de un "filtro invertido cargado" -materiales gruesos arriba- de forma que su peso, colocado en la zona donde emergen las líneas de corriente en forma concentrada, evite el sifonamiento e incremente el factor de seguridad [8].

En la figura 3 y la figura 4 se muestran socavones producto del fenómeno de colapso por humedecimiento. 


\section{investigación}

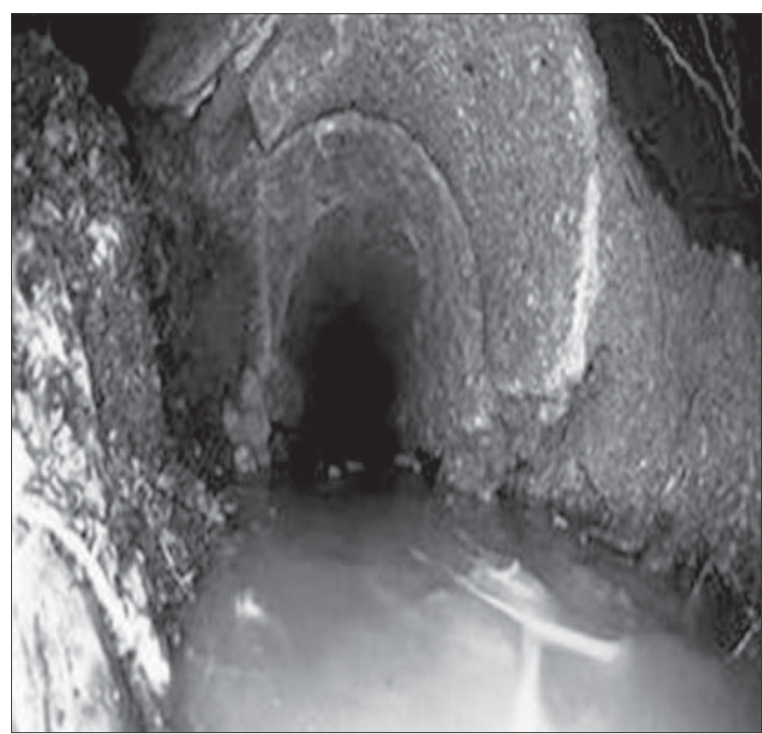

Figuras 3. Socavones producto del fenómeno de colapso por humedecimiento

Fuente: elaboración propia

\subsection{Exploración del subsuelo}

Los trabajos de muestreo de suelos tienen por objeto obtener la información necesaria para conocer los aspectos de los depósitos de suelos, tales como: estratigrafía del sitio, clasificación geotécnica de los suelos que forman cada estrato, compacidad relativa o consistencia de cada tipo de suelo identificado en el perfil estratigráfico, resistencia al esfuerzo cortante.

Con el fin de determinar las condiciones geotécnicas del sitio de estudio, se realizaron visitas de campo a diferentes sitios, ubicados en la $\mathrm{ZMG}$, observándose fracturas en la carpeta asfáltica así como cuarteaduras en estructuras, lo cual indica la presencia de suelos colapsables. Tomando en cuenta lo anterior, y con el objetivo de tener un marco de referencia que permita comparar su comportamiento de acuerdo con sus propiedades mecánicas y cumplir con el objetivo de esta investigación, se seleccionaron varios sitios para el estudio de esta zona (tabla 1). La figura 5 muestra la localización de los sitios de muestreo.

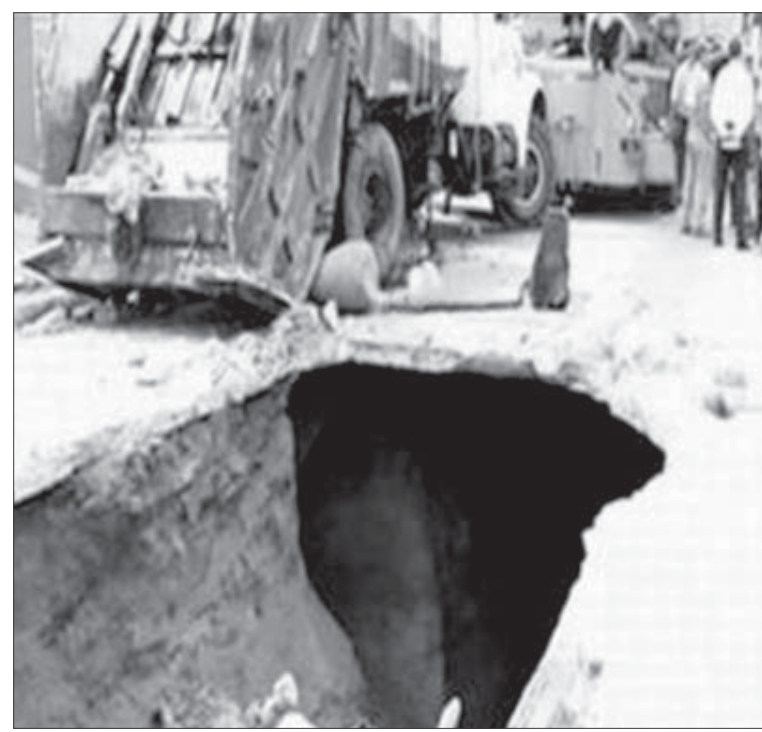

Figuras 4. Socavones producto del fenómeno de colapso por humedecimiento

Fuente: elaboración propia.

Los cortes observados en las figuras 6 y 7 muestran los materiales típicos del subsuelo de la Zona Metropolitana de Guadalajara, en los que se observan los estratos de arena y grava que están constituidos predominantemente por material pumítico.

Tabla 1. Localización de sitios de muestreo

\begin{tabular}{|c|c|}
\hline Localización & Tipo de muestra obtenida \\
\hline $\begin{array}{c}\text { Anillo Periférico Norte y Calzada } \\
\text { Independencia. (Norte de la ZMG) }\end{array}$ & Pozo a cielo abierto l. \\
\hline $\begin{array}{c}\text { Av. Adolfo López Mateos Sur y Av. de las } \\
\text { Rosas. (Sur-Oeste de la ZMG) }\end{array}$ & Pozo a cielo abierto 2. \\
\hline $\begin{array}{c}\text { Calzada Lázaro Cárdenas, (Sur-Oeste de la } \\
\text { ZMG ) }\end{array}$ & Pozo a cielo abierto 3. \\
\hline $\begin{array}{c}\text { Fraccionamiento Jardin Real.(Nor-Oeste de la } \\
\text { ZMG) }\end{array}$ & Pozo a cielo abierto 4. \\
\hline $\begin{array}{c}\text { Fraccionamiento Valle Real. (Nor-oeste de la } \\
\text { ZMG) }\end{array}$ & Pozo a cielo abierto 5. \\
\hline $\begin{array}{c}\text { Av. Americas y R. de Janeiro (Nor-oeste de la } \\
\text { ZMG) }\end{array}$ & Pozo a cielo abierto 6. \\
\hline $\begin{array}{c}\text { Diversos bancos de materiales alrededor de la } \\
\text { periferia de la ZMG }\end{array}$ & Pozo a cielo abierto. \\
\hline
\end{tabular}

Fuente: elaboración propia. 


\section{investigación}

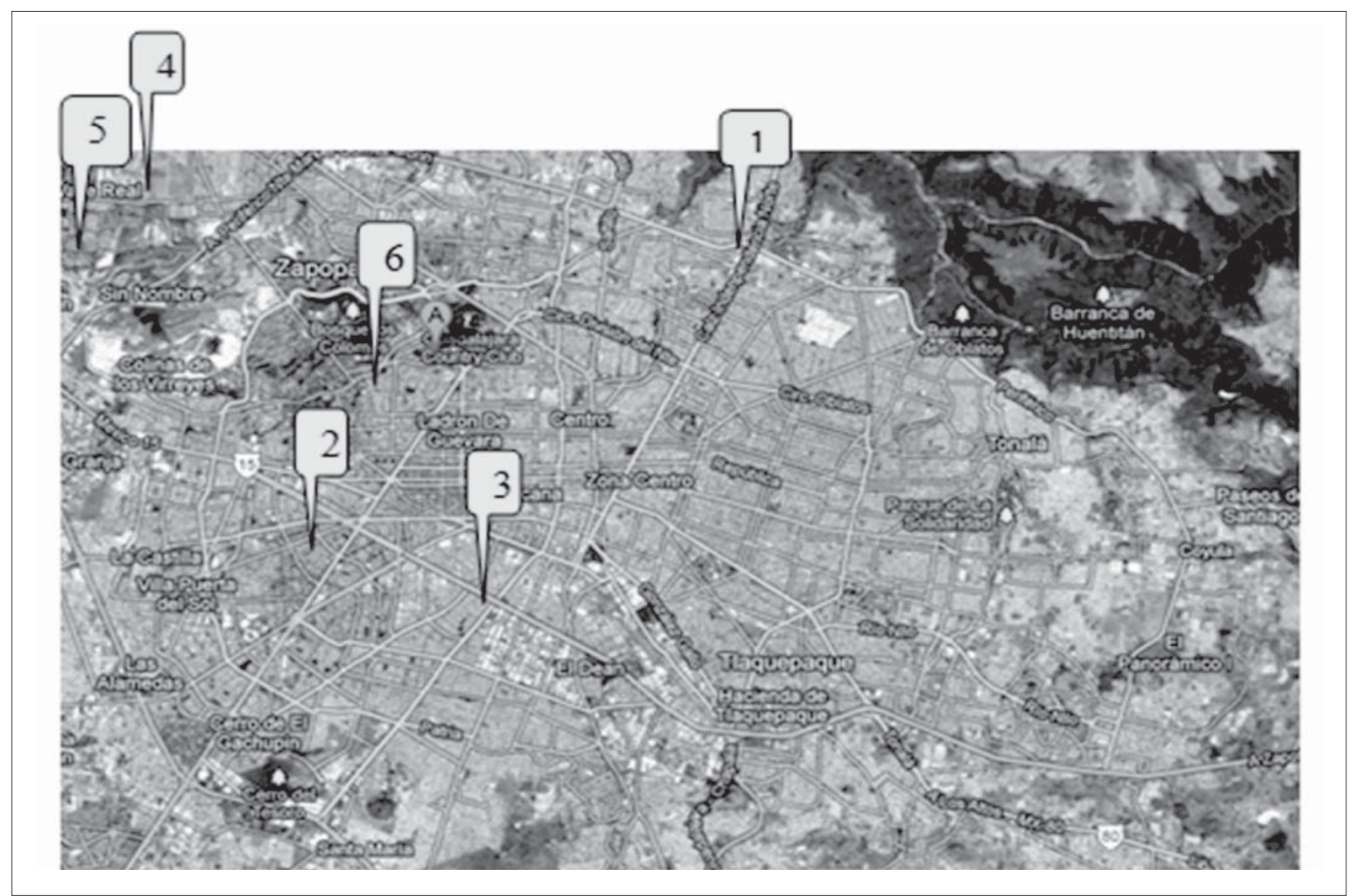

Figura 5. Localización de los sitios de muestreo

Fuente: elaboración propia.

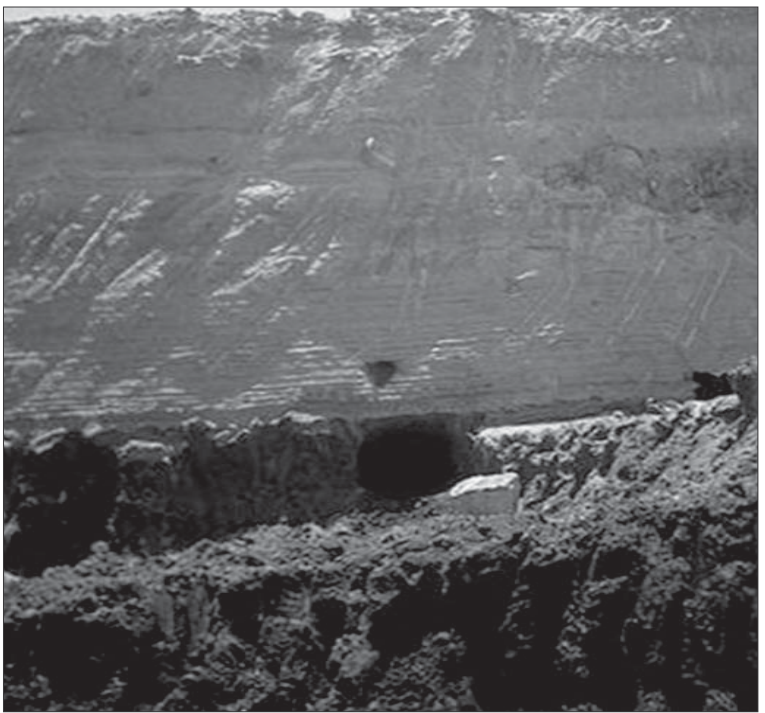

Figuras 6. Vista de diferentes cortes donde se obtuvieron muestras de suelo

Fuente: elaboración propia.

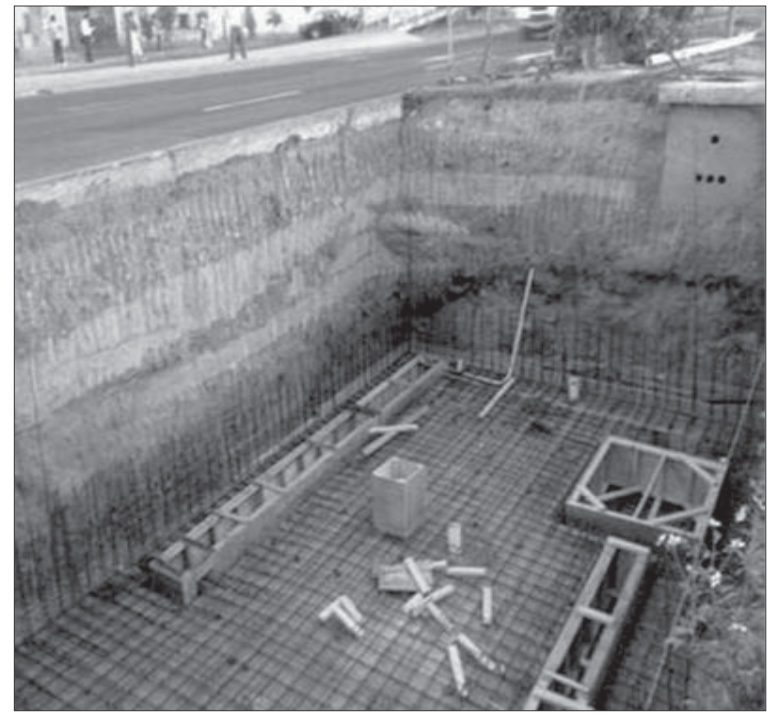

Figuras 7. Vista de diferentes cortes donde se obtuvieron muestras de suelo

Fuente: elaboración propia. 


\section{investigación}

Dicho trabajo constó en realizar un sondeo de pozo a cielo abierto (PCA) en los sitios de estudio, obteniendo solamente en tres de ellos muestras cúbicas, debido al tipo de material de los otros sitios, las muestras cubicas fueron obtenidas entre 1 y 6 metros de profundidad. Dichas muestras fueron de $40 \times 40 \mathrm{~cm}$ para obtener material que permita labrar las probetas, el material fue cubierto con manta de cielo y recubierto con brea y parafina, con el fin de protegerlo y conservar su contenido natural de humedad. Las muestras alteradas fueron obtenidas por medio de una pala, procurando que con el impacto no se rompieran los granos de material. Dicho material se colocó en costales para ser transportado al laboratorio.

También se ejecutaron sondeos de penetración estándar (SPT). Con el objetivo de determinar las condiciones estratigráficas particulares, sus propiedades índice y mecánicas, para caracterizar las condiciones del subsuelo requeridas para llevar a cabo el diseño constructivo de camas drenantes.

\subsection{Identificar los estratos de suelo colapsable, realizando pruebas índice}

El suelo de la zona en estudio está constituido, en casi la totalidad de la ciudad, de suelos pumiticos con granulometrías comprendidas en el rango de limos a gravas -llamadas localmente jales-, abundando principalmente arenas amarillas y arenas limosas. Las componentes de los suelos casi siempre van de altas a bajas conforme se va profundizando observándose un contraste marcado entre los depósitos poco compactos y compactos, hasta llegar a la roca basal. La figura 8 presenta una columna estratigráfica típica de los suelos de la ZMG.

El suelo donde se produce el fenómeno de colapso es en la arena limosa, cuyas características se describen en la tabla 2, la tabla 3 y la tabla 4.

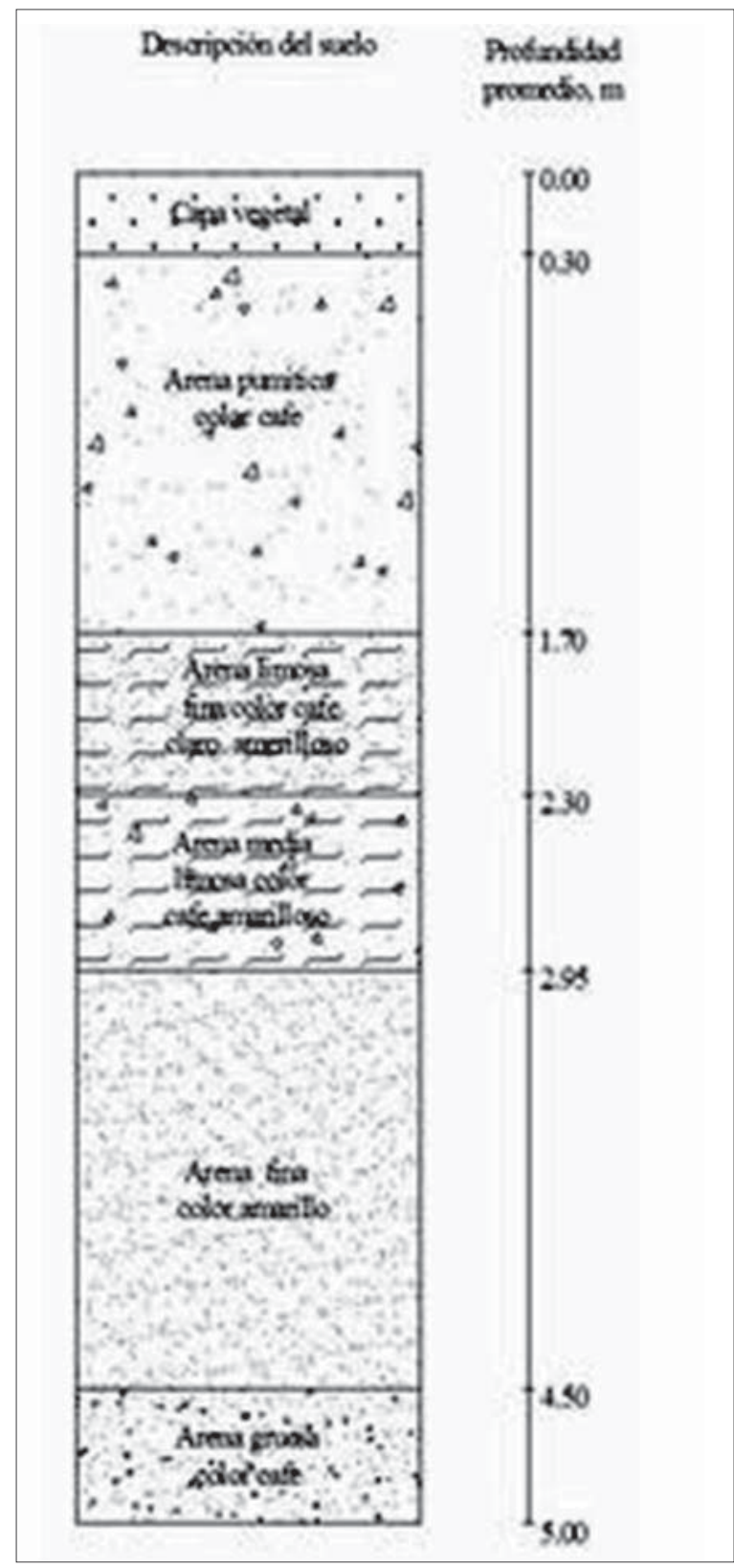

Figura 8. Columna estratigráfica típica de la ZMG

Fuente: elaboración propia.

\subsection{Simulación del suelo colapsable, en un modelo a escala.}

Se realizó una simulación a escala del fenómeno de tubificación, para ello se elaboró un cajón metálico (figura 9), el cual se llenó de arena limo- 


\section{investigación}

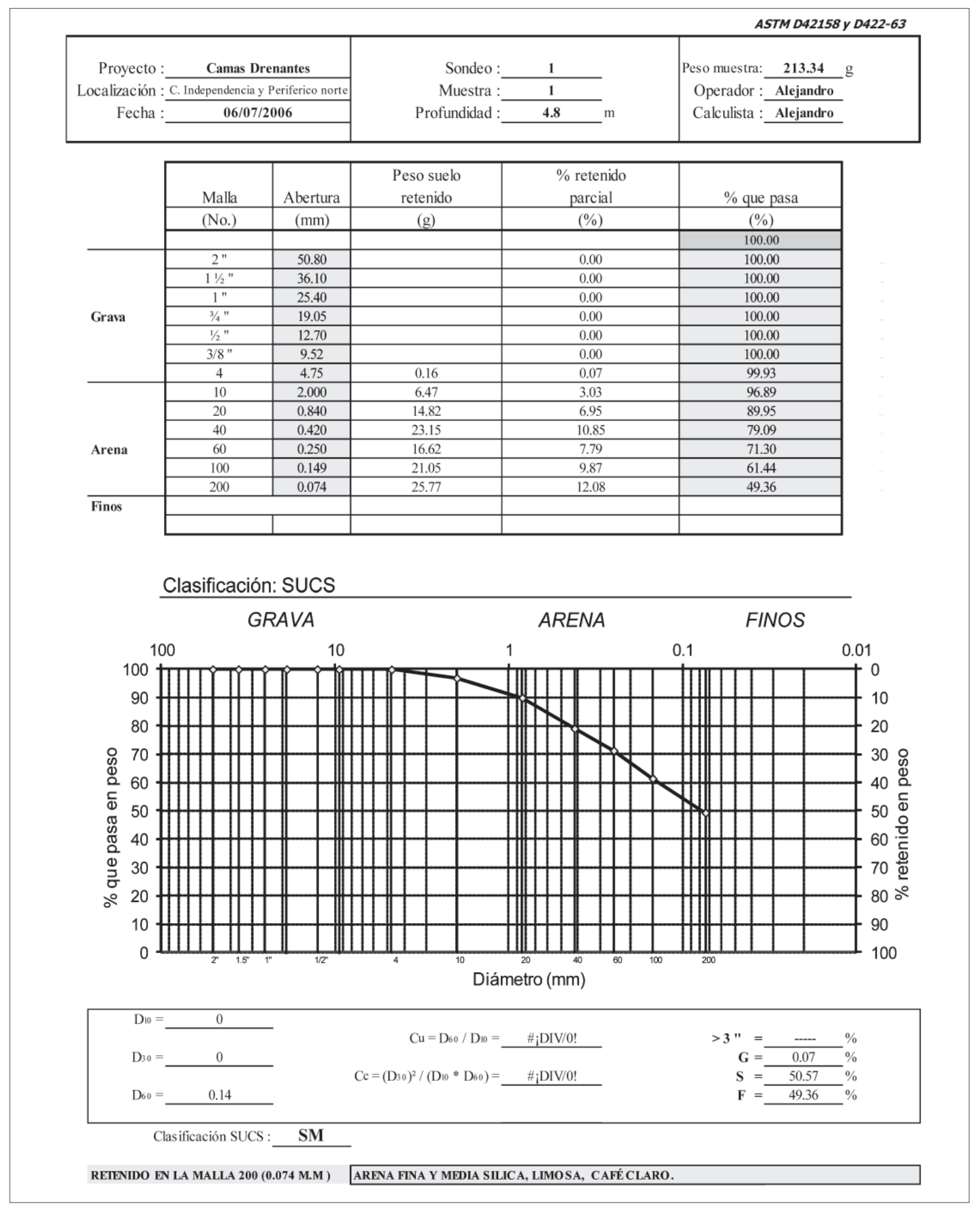

Tabla 2. Granulometría

Fuente: elaboración propia 


\section{investigación}

sa, suelo sobre el que se encuentran las líneas de drenaje de la zona en estudio; además, contenía una tubería perforada al centro de dicho cajón simulando una fuga (figura 10). En la parte central inferior del cajón se dejaron dos aberturas simulando dos medios discontinuos, la tubería contó con una pendiente del $2 \%$, se hizo pasar agua por la tubería para después empezar a filtrarse al suelo de apoyo, este se humedeció hasta llegar a saturarse, produciendo en consecuencia el fenómeno de colapso.

\subsection{Análisis de las características geotécnicas de la arena pumitica a emplear como dren.}

Se realizaron los ensayos a la arena pumitica con el objetivo de encontrar las mejores condiciones de permeabilidad y resistencia. Para ello, se realizaron diferentes combinaciones granulmetricas, observando que, entre mayor era el porcentaje del tamaño de los granos (tamaño de 4,75 mm a 12,7 $\mathrm{mm})$ y debido a su porosidad, tenia una mejor

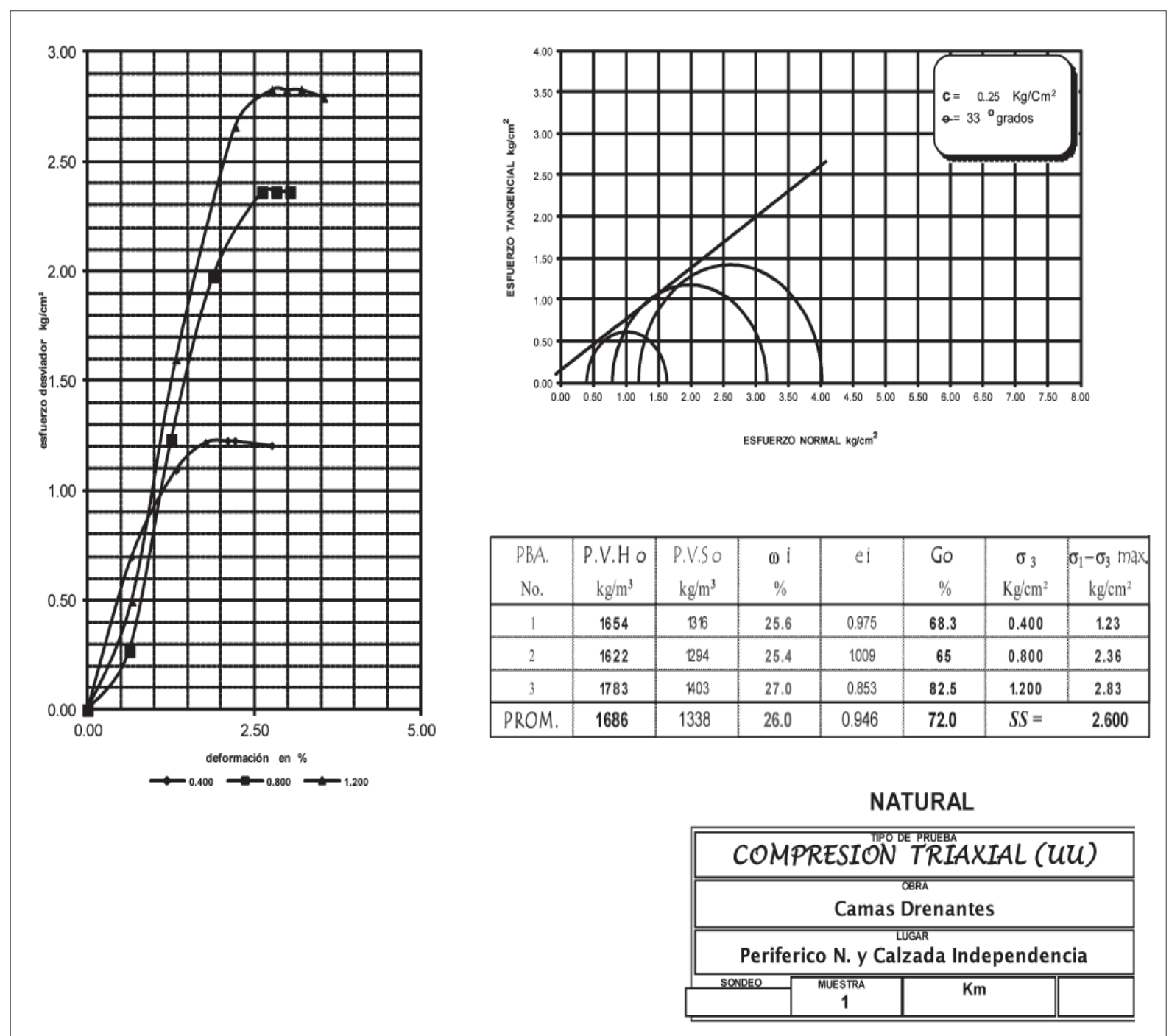

Tabla 3. Resistencia en estado natural

Fuente: elaboración propia 


\section{investigación}

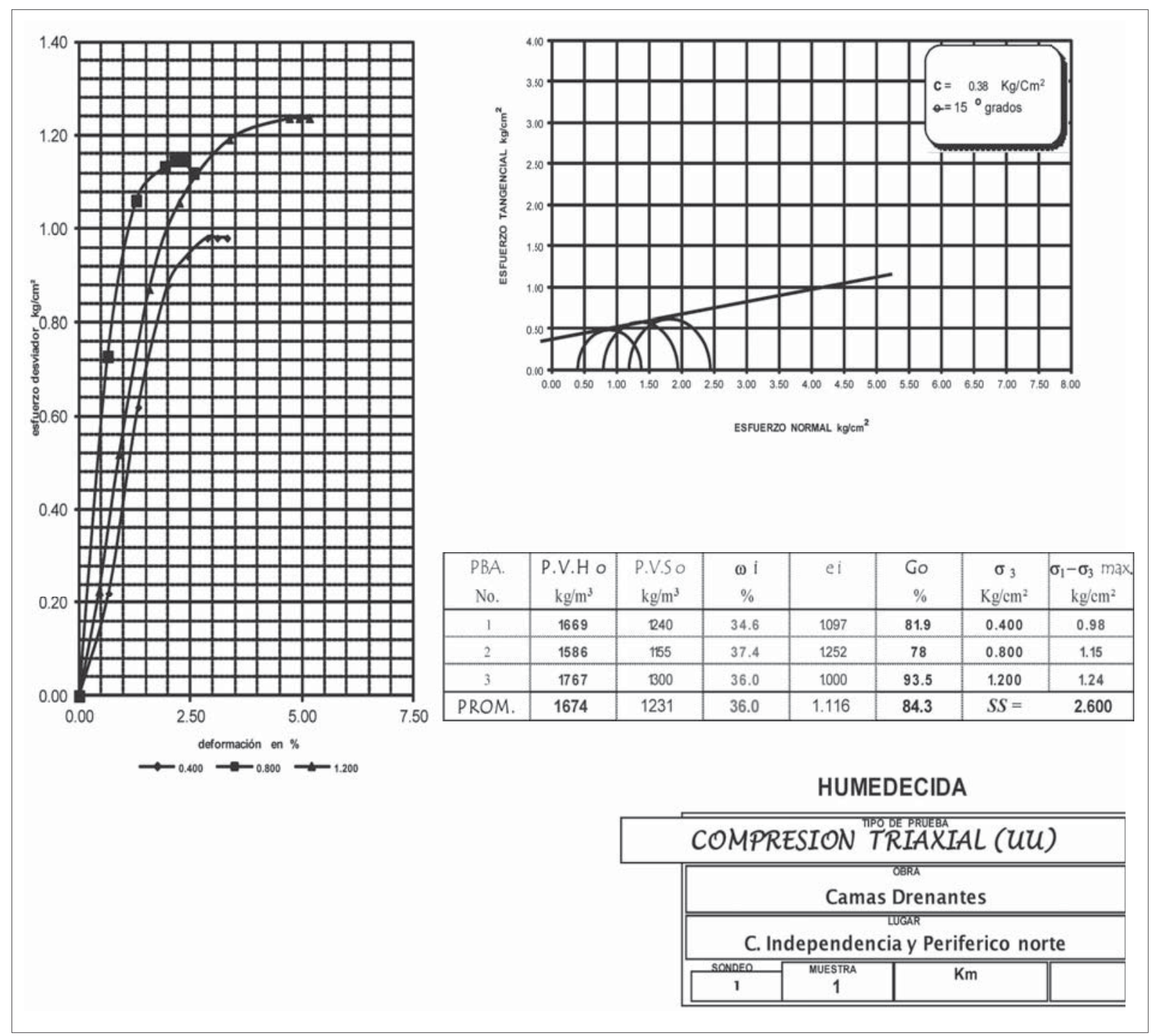

Tabla 4. Resistencia en estado saturado

Fuente: elaboración propia

permeabilidad y menor resistencia, en comparacion a si el porcentaje era mayor de particulas pequeñas (tamaño de $0,25 \mathrm{~mm}$ a $0,84 \mathrm{~mm}$ ). Se realizaron los ensayos hasta encontrar una granulometria que cumpliera con un equilibrio en la permeabilidad y resistencia, teniendo en cuenta que fuera una granulometria fácil de encontrar en la mayor parte de la ZMG y los bancos de material cercanos a la periferia de la ciudad. En la figura 11 se muestra el equipo utilizado.
Las características del suelo a emplear se describen en la tabla 5 .

Los resultados obtenidos se comprobaron en el mismo modelo a escala que se utilizó para el fenómeno de colapso como se muestra en la figura 12. Empleando en la parte inferior una capa de polietileno para evitar que el flujo pase al suelo inferior y canalizarlo todo por el dren logrando el resultado esperado. 


\section{investigación}

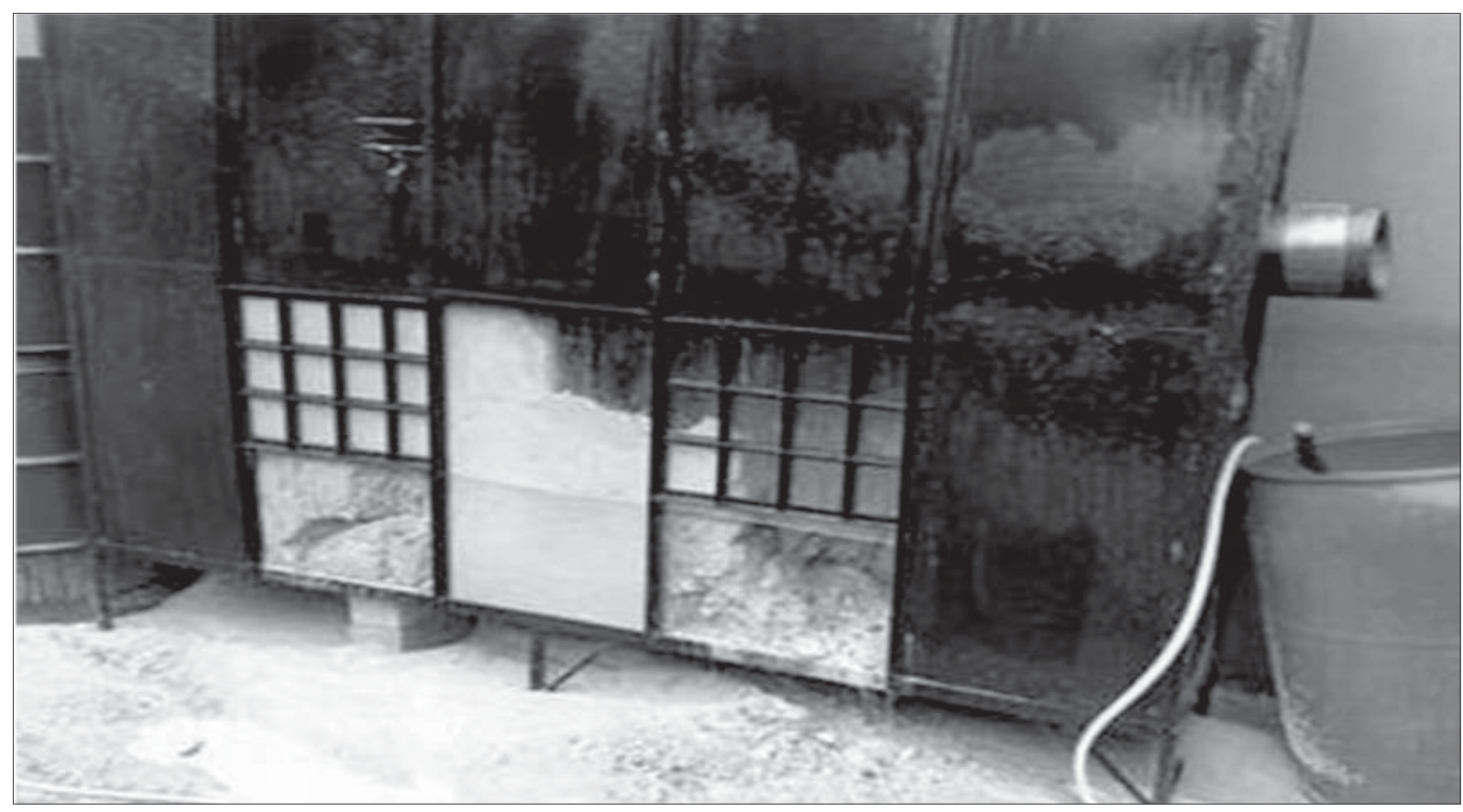

Figura 9. Modelo a escala

Fuente: elaboración propia
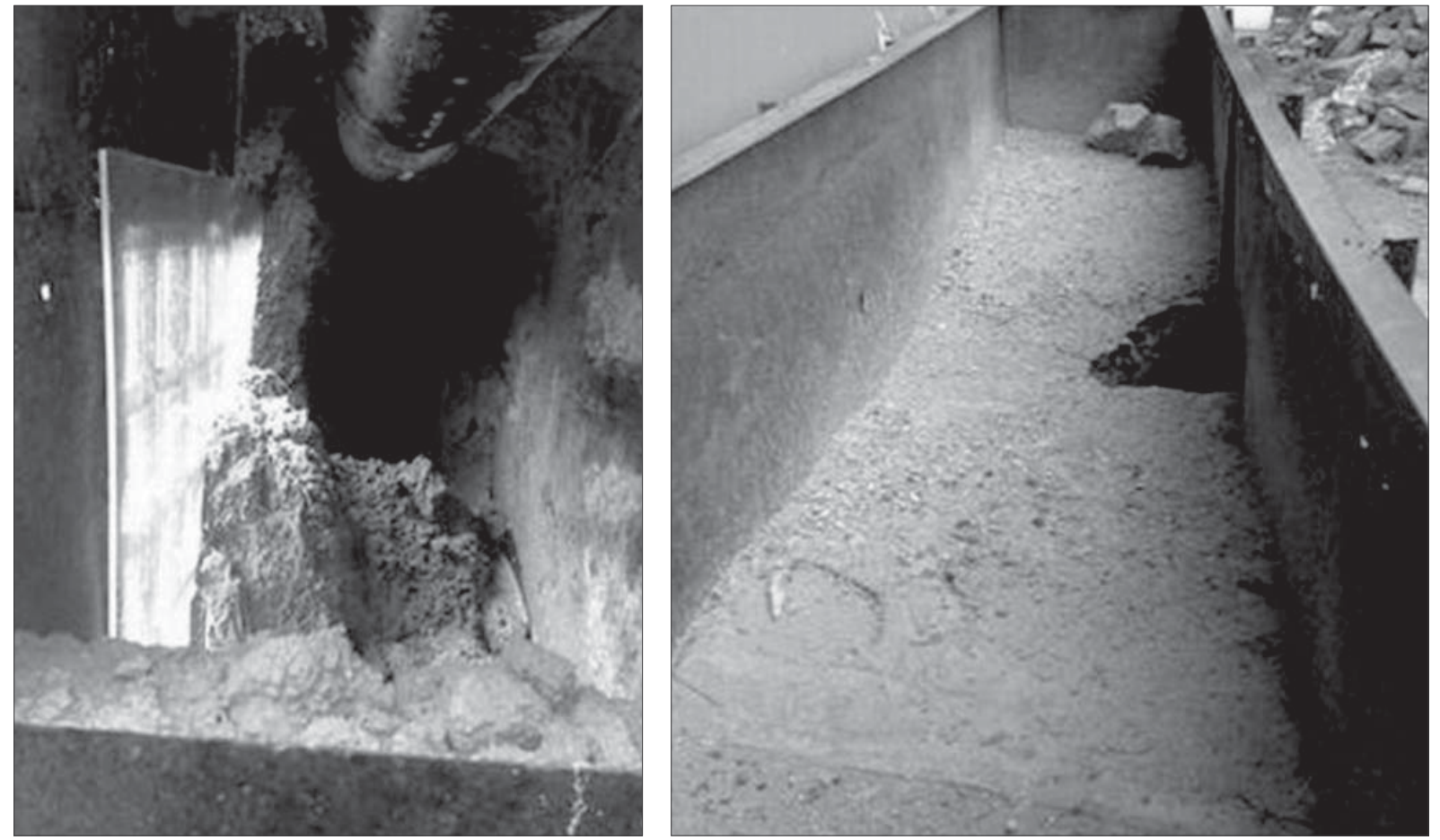

Figura 10 Fenómeno de colapso

Fuente: elaboración propia. 


\section{investigación}
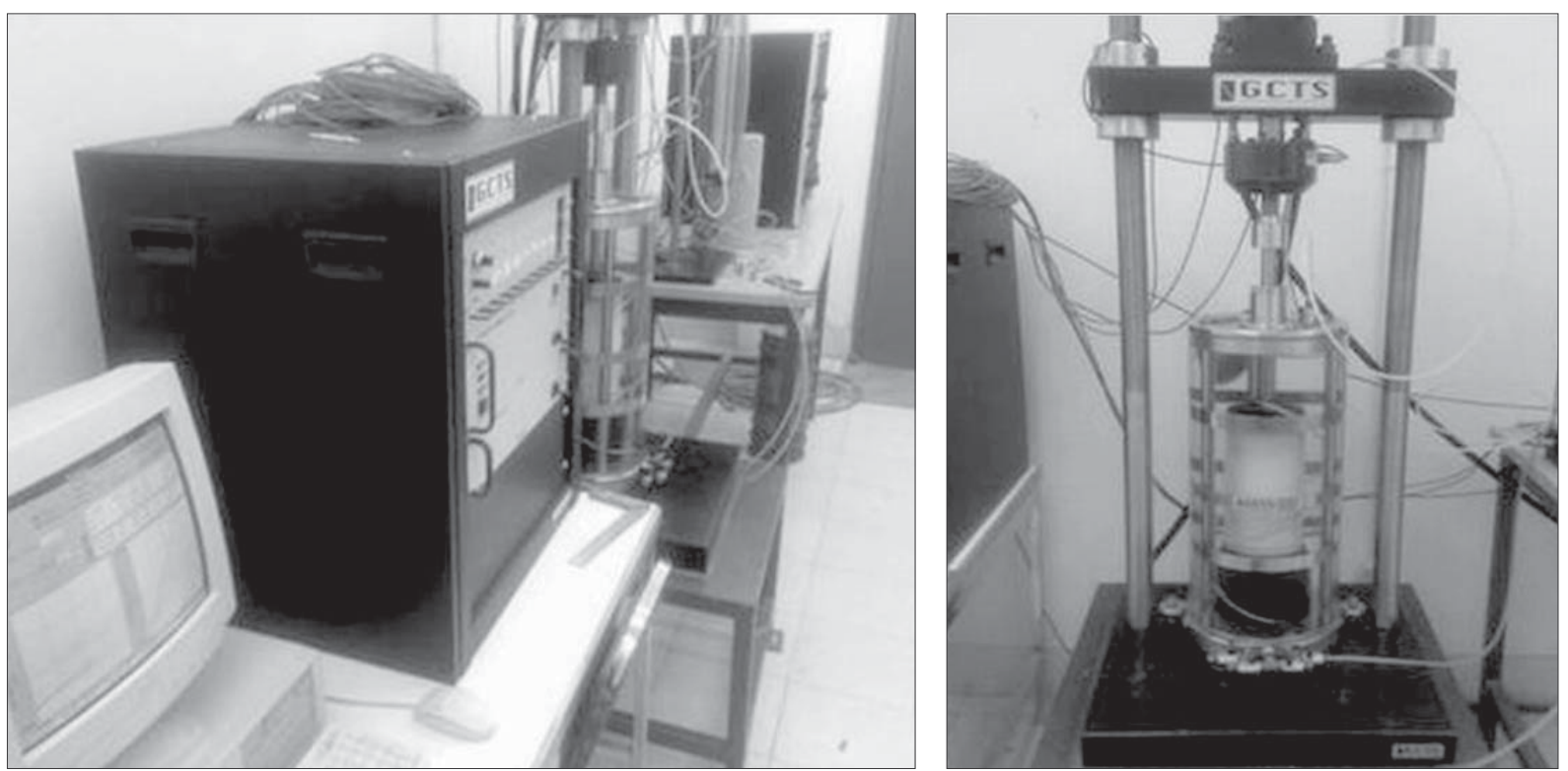

Figura 11. Equipo triaxial utilizado

Fuente: elaboración propia.

Tabla 5. Características de la cama drenante

\begin{tabular}{|c|c|c|c|c|}
\hline Muestra & $\begin{array}{c}\mathbf{g} \\
(\mathbf{g} / \mathbf{c m} 3)\end{array}$ & $\mathbf{w}(\%)$ & $\boldsymbol{\varphi}\left(^{\circ}\right)$ & $\mathbf{K}(\mathbf{c m} / \mathbf{s})$ \\
\hline Final & 1,25 & 32 & 39 & 0,108 \\
\hline
\end{tabular}

Fuente: elaboración propia.
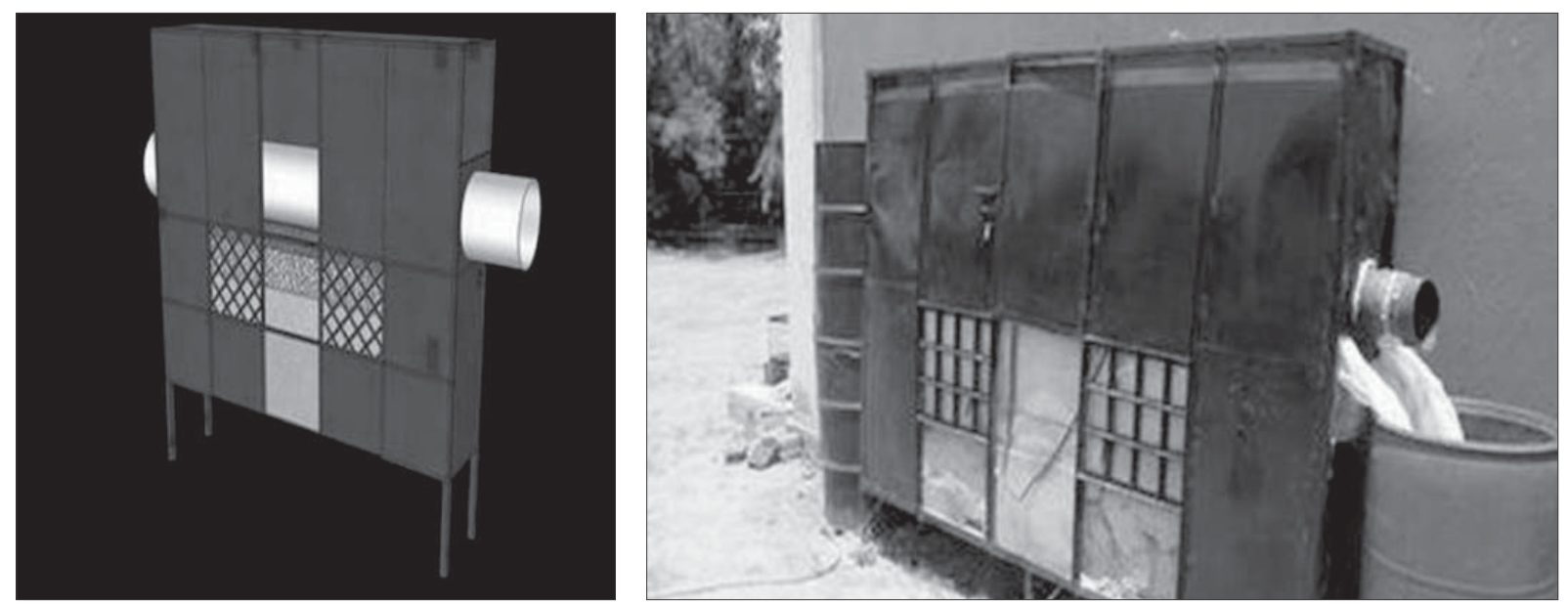

\section{CONCLUSIONES Y RECOMENDACIONES}

En base a la investigación realizada de suelos colapsables, se observó que la arena limosa, sobre la que se encuentran las líneas de drenaje, tiende al colapso por dos situaciones: la primera, por

Figura 12 Modelo a escala con cama drenante Fuente: elaboración propia. 


\section{investigación}

humedecimiento la cual genera que el suelo pierda más de la mitad de su resistencia; y la segunda, como se representó en el modelo a escala, es cuando se encuentra un medio discontinuo cercano a la zona del suelo que se encuentra saturado, ocasionando el fenómeno de tubificación el cual arrastra las partículas más pequeñas produciendo un reacomodo de las partículas en el área afectada, dando origen a los socavones que afectan las vialidades y construcciones en la ZMG.

Las características de la arena pumitica que se encuentra en gran parte de la ciudad cumple adecuadamente para formar parte de un dren que se emplee debajo de las líneas de drenaje que sean restauradas o nuevas. Este dren debe estar envuelto en una capa de polietileno que impida el flujo vertical y, por lo tanto, sea de manera horizontal. Esta modificación al sistema constructivo de las líneas de drenaje conlleva realizar una excavación de $20 \mathrm{~cm}$ más de lo proyectado, esto para instalar la cama drenante, dicho espesor será capaz de ayudar a desalojar el flujo producto de una fuga al siguiente pozo de visita.
Con la modificación e implementación mencionada, se evitará que, si ocurre una fuga, haya consecuencias en la estabilidad del suelo de apoyo y, además de evitar la contaminación del subsuelo, dará un tiempo necesario para que el ayuntamiento pueda reparar dichas fallas sin consecuencia alguna.

\section{FINANCIAMIENTO}

Esta investigación forma parte de la tesis de grado de un servidor para obtener el título de maestro en ciencias del Instituto Politécnico Nacional, y se contó con el apoyo en el uso del equipo de laboratorio del Instituto de Ingeniería Sísmica de la Universidad de Guadalajara.

\section{AGRADECIMIENTOS}

A la escuela superior de Ingeniería y Arquitectura del Instituto Politécnico Nacional por su asesoría. Al departamento de Ingeniería civil y topografía de la Universidad de Guadalajara.

\section{REFERENCIAS}

[1] E. Redolfi, "Suelos Colapsables". Universidad Nacional de Córdoba, Córdoba. 2007. Disponible: http://www.docentes. unal.edu.co/aepazgon/docs/Suelos $\% 20$ Cola psables.pdf

[2] J. Jimenez, "Introducción a los fenómenos de expansividad y colapso en suelos y rocas", Apuntes del curso sobre cimentaciones en terrenos metaestables: colapsables y expansivos, Vol 1. Ed. Fundación Agustín de Bethencourt. E.T.S. de Ingenieros de caminos, canales y puertos. Universidad Politécnica de Madrid.1986.

Tecnura | Vol. 17 | Número Especial | pp. 79 - 92 | Julio de 2013
[3] A. Reginatto and J. Ferrero, "Collapse potencial of soils and soil water chemistry", Proc. of the octave international conference on soil mechanics and foundation engineering, Moscu, URSS. Vol 2, 2, 1973 pp. 177-183.

[4] A. Reginatto, "Predicción de la susceptibilidad al colapso", Segunda reunión argentina de mecánica de suelos e ingeniería de fundaciones, Córdoba, Argentina. 1970.

[5] R. Rocca, "Review of engineering properties of loess", Submitted for completion of 
requirements for the degree of master of engineering in geotechnical engineering, University of California, Berkeley. Report CE 299. 1985.

[6] E. Redolfi, R. Rocca y R. Terzariol, "Estudio comparativo de diferentes métodos para evaluar el potencial de colapso en suelos loésicos Argentinos", Memorias del simposio Argentino de suelos colapsables, Buenos Aires, Argentina. pp. 46-66, 1986.
[7] G. Aitchison. "Structurally Unstable Soils. State of the Arts". Proc of the $8^{\circ}$ International Conference on Soils Mechanics and Foundation Engeneering, vol 3, 1973 pp. $161-190$,

[8] K. Terzaghi y R. Peck. Mecánica de suelos en la ingeniería práctica, Buenos Aires: El Ateneo, 1996. 